\title{
Comparative effectiveness of hand scaling by undergraduate dental students following a two-week pre-clinical training course
}

\author{
Gartenmann, S J ; Hofer, D ; Wiedemeier, D ; Sahrmann, P ; Attin, T ; Schmidlin, P R
}

\begin{abstract}
BACKGROUND The Bologna reform resulted in a drastic restructuring of pre-clinical training courses at the University of Zurich. The aim of this study was to assess student pre-clinical scaling/root planning skills after just 8.5 hours of manual training. MATERIAL AND METHODS Three consecutive classes of dental students $(\mathrm{n}=$ $41 ; \mathrm{n}=34 ; \mathrm{n}=48$ ) were tasked with removing lacquer concrement from the maxillary left canine on a typodont using Gracey and universal (Deppeler M23A) curettes. At baseline (prior to instruction), a timed five-minute session of scaling/root planning was undertaken. The second scaling/root planning session was held immediately following training. Eight experienced dental hygienists and eight lay people served as positive and negative controls, using the same instruments and time limit, respectively. Instrumented teeth were collected, scanned and planimetrically analysed for the percentage of tooth surface cleaned. Statistical analyses were performed to assess the dental students' improvement after the training (Wilcoxon signed-rank test) and to compare it to that of laypeople and dental hygienists (Kruskal-Wallis rank sum test followed by Conover's post hoc test). RESULTS At baseline, the dental students' mean scaling scores of the cleaned surfaces were not significantly different than those of laypeople $(29.8 \%, 31.0 \%, 42 \%$ vs $27.9 \%)$. However, after 8.5 hours of manual training, the students' ability to clean the maxillary tooth improved significantly and they achieved mean removal values of $61.7 \%, 79.5 \%$ and $76 \%$ compared to the $67.4 \%(\mathrm{P}<.001)$ of the experienced dental hygienists (Tables Tables and ). There were no statistically significant differences between the scores achieved by students after training and those achieved by experienced dental hygienists. CONCLUSION A shortened pre-clinical training time was sufficient for students to acquire the basic scaling/root planning skills needed in preparation for clinical training. Further research is needed to identify ways to help students consistently reach highest skill levels.
\end{abstract}

DOI: https://doi.org/10.1111/eje.12361

Posted at the Zurich Open Repository and Archive, University of Zurich

ZORA URL: https://doi.org/10.5167/uzh-167390

Journal Article

Accepted Version

Originally published at:

Gartenmann, S J; Hofer, D; Wiedemeier, D; Sahrmann, P; Attin, T; Schmidlin, P R (2019). Comparative effectiveness of hand scaling by undergraduate dental students following a two-week pre-clinical training course. European Journal of Dental Education, 23(1):1-7.

DOI: https://doi.org/10.1111/eje.12361 


\title{
Comparative effectiveness of hand scaling by undergraduate dental students following a two-week pre- clinical training course
}

\author{
S.J. Gartenmann ${ }^{\text {a }}$, D. Hofer ${ }^{\text {a, }}$ D. Wiedemeier ${ }^{\text {b }}$, P. Sahrmann ${ }^{\text {a }}$, T. Attin ${ }^{\text {a }}$, \\ P.R. Schmidlin ${ }^{a}$
}

a Clinic of Preventive Dentistry, Periodontology and Cariology, Center of Dental Medicine, University of Zurich, Switzerland

b Statistical Services, Center of Dental Medicine, University of Zurich, Switzerland

Address for correspondence:

Prof. Dr. Patrick R. Schmidlin

Clinic of Preventive Dentistry, Periodontology and Cariology

Center of Dental Medicine, University of Zurich

Plattenstrasse 11

$\mathrm{CH}-8032$ Zurich

Phone: +41446343417

Fax: $\quad+41446344308$

e-mail: patrick.schmidlin@zzm.uzh.ch

Running title: Dental students' pre-clinical scaling competency

Keywords: dental students, dental education, pre-clinical competency, scaling and root planing, motor skills

Conflict of interest and source of funding statement No external funding was received for this study and none of the authors report any conflict of interest. 


\section{Abstract}

Background: The Bologna reform resulted in a drastic re-structuring of pre-clinical training courses at the University of Zurich. The aim of this study was to assess student pre-clinical scaling/root planing skills after just 8.5 hours of manual training.

Material and Methods: Three consecutive classes of dental students $(n=41 ; n=$ $34 ; n=48$ ) were tasked with removing lacquer concrement from the maxillary left canine on a typodont using Gracey and universal (Deppeler M23A) curettes. At baseline (prior to instruction), a timed 5-minute session of scaling / root planing was undertaken. The second scaling/root planing session was held immediately following training. Eight experienced dental hygienists and eight laypeople served as positive and negative controls, using the same instruments and time limit, respectively. Instrumented teeth were collected, scanned and planimetrically analyzed for the percentage of tooth surface cleaned. Statistical analyses were performed to assess the dental students' improvement after the training (Wilcoxon signed-rank test), and to compare it to that of laypeople and dental hygienists (Kruskal-Wallis rank sum test followed by Conover's posthoctest).

Results: At baseline, the dental students' mean scaling scores of the cleaned surfaces were not significantly different than those of laypeople $(29.8 \%, 31.0 \%, 42 \%$ vs. $27.9 \%$ ). However, after 8.5 hours of manual training, the students' ability to clean the maxillary tooth improved significantly and they achieved mean removal values of $61.7 \%, 79.5 \%$ and $76 \%$ compared to the $67.4 \%(p<0.001)$ of the experienced dental hygienists (Tables 4 and 5). There were no statistically significant differences between the scores achieved by students after training and those achieved by experienced dental hygienists.

Conclusion: A shortened pre-clinical training time was sufficient for students to acquire the basic scaling / root planing skills needed in preparation for clinical training. Further research is needed to identify ways to help students consistently reach highest skill levels. 


\section{Introduction}

The European education system underwent a deliberate and major overhaul in the early part of the 21st Century, beginning with the Bologna Declaration of 1999 (1). European Universities were challenged to restructure their national educational programs to create and then comply with a new set of goals, whose aim was to create a "European identity" in higher education (2). Broad strokes were laid out, which were at the same time both minimum requirements to be retained from the "old" national curricula and guidelines for modernizing the learning process, which would better support the implementation of best practices as they emerge.

This change in educational focus was especially challenging in the dental school curriculum, as not only is academic excellence required to successfully complete this course of education, but also the acquisition of high-level manual skills. In an effort to comply with a major goal of the new system, the introduction of a bachelor degree Europe-wide, the dental school curriculum in Zurich was trimmed of some pre-clinical and clinical practice time, to make room for self-study, self-exploration and integrative learning mandated under the new "Bologna system" curriculum.

The ultimate goal of the dental education curriculum at the University of Zurich remained the education of clinical practitioners who could both competently treat dental disease and implement preventive strategies for the good of the local population, while adding on the skills needed to find, analyze, evaluate and integrate new information and techniques. The faculty was tasked with developing new teaching techniques that would impart the traditional knowledge and skills training in a shorter time period than had been the case under the previous system. The overall educational time frame was kept at five years, even as new themes were added to the curriculum.

In this context, the pre-clinical scaling/root planing skills lab was reduced from one 
semester of instruction and practice (appr. 60 hours) to a two-week course of 32 hours in total. Eight and a half hours of manual training on mannequins were combined with 15 hours of theory and five hours of student presentations (with the remaining time consumed with course administration, set up, clean up and a daily 20 minute break). Dental skills are not in-born, but rather acquired through exposer to and the practice of different tasks. Hence, we investigated the development of manual dexterity skills of dental students entering a pre-clinical course, which would prepare them for treating patients and giving them a solid basis for further developing the manual skills necessary to successfully instrument more complex cases. Successful completion of this course is required before progressing to the clinical part of their dental education, treating periodontally involved patients, using the same techniques practiced on the colored lacquer-covered teeth. While the students were evaluated empirically as having learned the necessary skills, it appeared expedient to quantify their capabilities and compare them with other clinicians who had received a classical training. The following questions aided as a guide to evaluate this experimental success. 1 . Is the time given for a periodontal pre-clinical class sufficient enough to teach the students techniques in periodontal instrumentation? 2. What level of efficiency can dental student achieve in two weeks?

Therefore, the purpose of this study was to identify the progress of dental students' motor skills on one single-rooted tooth during an intensive two-week preclinical periodontal course.

\section{Material and Methods}

\section{Ethic considerations}

The local ethic review board reviewed the study (BASEC-Nr. Req-2017-00492) and provided a certificate of non-objection. All student participants in this study were 
informed of the purpose and scope of the study prior to expressing their willingness to take part in the evaluation of their scaling / root planing skills. The participants were assured that the data collected would be anonymized and specifically for the dental students, should they elect to not take part in the study, that there would be no prejudice in terms of grading for the periodontal skills lab. Further, no compensation was offered for participation in this study.

\section{Operators}

One hundred twenty three dental students of three consecutive years (2014: $n=41$; 2015: $n=34 ; 2016: n=48$ ) volunteered to be evaluated for their scaling/root planing abilities before and after a pre-clinical skills course. This represents all students enrolled in their first year of dental school during the study period of 2014 through 2016. The same test was repeated for each class during the 3-year period. Prior to the pre-clinical training course, the students had neither been exposed in the curriculum to Gracey curettes, universal scalers nor worked on the periodontal typodont. A negative control group $(n=8)$ was comprised of laypeople and represented a broad spectrum of occupations such as office clerks, facility managers and a nurse to name a few. All laypeople that were asked to participate on a voluntary basis were either employed by the dental clinic or the university hospital and had no in-depth knowledge in dentistry. To build a similarly-sized positive control group, dental hygienists $(n=8)$ working at the dental school were asked to participate. The dental hygienists were employed in different departments, were available on the day of study and volunteered to participate. 


\section{Hand held Instruments}

Four commonly used site-specific Gracey curettes (11/12, 13/14, 7/8 and 5/6;

Deppeler SA, Rolle, Switzerland) and one universal curette (M23A; Deppeler SA, Rolle, Switzerland) were used in this study. The instruments were either new or adequately sharpened before each experiment; both before and upon completion of the course.

\section{Typodont model}

A model of the maxilla (Frasaco $\mathrm{GmbH}$, Tettnang, Germany) made out of hard thermosetting plastic material and covered with a fixed elastic gingiva was used for this study and is the traditional maxillary teaching model for scaling / root planing in Zurich. The model simulates periodontitis in its early to advanced stages and includes periodontal probe readings ranging between $3-9 \mathrm{~mm}$. The maxillary left canine is designed with a mesial horizontal defect of $6 \mathrm{~mm}$ and a PPD of $7 \mathrm{~mm}$ palatinal. The facial aspect presents with a Stillman's cleft of $8 \mathrm{~mm}$ from the cemento-enamel junction to the gingival margin and $1 \mathrm{~mm}$ PPD. This canine was designated as the study tooth and was coated from approximately $5 \mathrm{~mm}$ above the cemento-enamel junction to the apex of the tooth with a black nail lacquer (Manhattan, COTY Germany GmbH, Mainz).

\section{Experimental procedure}

Upon entering the skills lab, the students were instructed to insert the maxillary typodont model into the mannequin head at their workstation. They were asked to unpack their new Gracey curettes and universal scaler (instructor sharpened). After a brief introduction on the cutting edge of the instruments, they were instructed to remove the black lacquer concrement from the study tooth, within a timed 5-minute 
period. These teeth were then removed from the typodonts and collected for evaluation (Fig. 1). No note was made of which tooth came from which student, to preserve anonymity. Replacement teeth were then distributed and the first official scaling practice session of the course commenced.

On the last course day, prior to their announced practical exam (scaling and root planing of one quadrant, for grading purposes), a new tooth, which had been lacquered as described above, was distributed for post-course scaling/root planing, over the same 5-minute time period, and collected anonymously once again for evaluation.

The positive and negative control groups, as described above, completed the same exercise on the same mannequin heads using (instructor sharpened) school instruments and typodonts, over the same 5-minute timed period. Whereas the positive control group, the dental hygienists, required no instruction on the use of the instruments, the negative control group where shown the cutting edges and then allowed to use the instruments as they saw fit.

A reference tooth debrided under optimum conditions (direct vision, without the model's gingival mask, Fig. 2) by the teaching team dental hygienist, served as a reference tooth for the planimetric evaluation.

\section{Evaluation of scaled teeth}

After each treatment, the collected study teeth were scanned (Hewlett Packard C1750A, Houston Texas, USA) using custom grey-scale recognition software and planimetrically compared to the reference tooth. To this end, the teeth were fixed on a REM carrier (Laubscher, Täuffelen, Switzerland) and positioned on a guide trail to generate a three-dimensional image. The images were digitalized and the percentage of surfaces with residual nail lacquer was measured planimetrically on 
the area from $5 \mathrm{~mm}$ above the cervix (area corresponding to the cement enamel junction) to the apex of the tooth. The mask of the reference tooth with a gray scale threshold of 157 served as a reference for the other test teeth. To obtain the percentage of cleaned surfaces, the grey scale areas were calculated and statistically analyzed.

\section{Statistical analysis}

All statistical analyses and plots were done with the statistical software $R(3)$ and the PMCMR package (4). Descriptive statistics such as means, standard deviations, medians and interquartile ranges of the cleaned surfaces, expressed as a percentage of the total surface area, were calculated. The improvements in student's scaling skills after each year's training were assessed using the Wilcoxon signedrank test. The Kruskal-Wallis test followed by Conover posthoc test with Holm adjustment for multiple testing was used to analyze differences in scaling skills between dental students, laypeople and dental hygienists. .

\section{Results}

At baseline, the mean scaling skills of dental students in the classes 2014 and 2015 showed no statistical significance to those of laypeople (29.8\% and $31.0 \%$ vs. 27.9 \%). Students in 2016 , with a mean of $42.1 \%$ of the surface cleaned at baseline, achieved slightly better results than the students in the pervious 2 years. However, their results were also not statistically better than the mean results of the laypeople (Tables 4 and 5). After 8.5 hours of manual scaling training on a typodont, the students were able to improve their scaling skills and achieved mean removal values of $61.7 \%$ in $2014,79.5 \%$ in 2015 and $76 \%$ in 2016 . On average, the students in the years 2015 and 2016 achieved significantly higher scores than the students in 2014 . 
Also higher scores, though not statistically significant, where achieved in 2015 and 2016 compared to the experienced dental hygienists (67.4\%; NS). The 2014 class score average, while lower than the following two classes, showed no statistical difference to the average score of the dental hygienists (Fig. 3). A score of $>90 \%$ cleaned surfaces, close to the reference tooth cleaned under direct vision $(96.9 \%)$, was observed on individual teeth by individual students in various classes. In 2014 one student, in 2015 six students and in 2016 seven students removed nearly all the nail lacquer from the tooth surfaces.

\section{Discussion}

This study investigated whether 8.5 hours of manual skills training in periodontal debridement (mechanical scaling/root planing) was sufficient for first year dental students to successfully remove simulated concrement on typodont teeth. Our hypothesis that these students would receive sufficient training with different instruments and usage techniques on typodonts, as compared to clinicians who had received a traditional semester-long training in periodontal instrumentation (and have had $\geq 2$ years post-graduate work experience), was corroborated by the results of this study. The shorter, intense 8.5 hour distributed throughout a 3-week instrumentation skills course provided the necessary instruction to prepare our firstyear dental students to begin clinical scaling / root planing treatment on patients suffering from periodontal disease. The quantitative results from this study also provided a confirmation of the traditional visual assessments made for the student typodonts by the teaching staff for grading purposes after full quadrant testing at the end of the course.

Traditional manual dexterity tests for applicants seeking admission to dental school were used for a long time in the hope of predicting clinical success (5-12). Most of 
these tests have been abolished, as their predictive values have provided only inconsistent results (13-15). Ranney et al. (16) suggest that perceptual ability or manual dexterity tests are best viewed as screening rather than predictive tools since there is evidence that dental skills can be learned during dental curricular education. This was further proven in a study done on 433 dental students tested in five consecutive years whereby basic manual dexterity scores improved with training in pre-clinical and clinical education (17). The test designed by the investigators entailed 10 exercises with specific materials and tasks. Students, although not informed of their mistakes, improved significantly, including students with initially weak manual abilities.

In our study groups, the consecutive dental student cohorts from 2014 to 2016 had identical training sessions and repeated the experiment under the same conditions. Delving further into the results presented, all three classes showed a normal distribution among the groups, as one would expect in a group of students learning a new skill set. There was more improvement seen after the training course for students in the years 2014 and 2015, and all three classes shortened the range of scores after completion of the instrumentation course.

The dental students also displayed abilities equal to or even slightly better by trend than the graduate dental hygienists after 8.5 hours of training when removing concrement from the artificial plastic teeth. Several studies have investigated the efficacy of scaling and root planing with inexperienced operators using various forms of instrumentation and tooth sites (18-22). Kocher and co-workers investigated the substance removal and debridement during simulated open flap operation on artificial teeth with curettes and modified sonic scaler tips (20). The six operators were of different experience levels: Periodontal residents with at least two years of experience and dental students. The goal of this study was to investigate the 
operators' success in removal of concrement based on the instruments used or their level of experience. The percentage of the debrided areas between the instrument types used showed little difference, whereas the differences between operators were statistically significant. The experienced operators obtained $85 \%$ concrement removal as compared to the dental students who successfully debrided $65 \%$ of the total surface. Further evaluation of the operators after training, however, was not pursued in this study. In comparison, the dental students in our study achieved surface debridement levels of $29.8 \%, 31.0 \%$ and $42.1 \%$ at baseline, respectively, under closed conditions. They improved their performance, however after training to $61.7 \%, 79.5 \%$ and $76 \%$, which represents higher scores than presented in the previous study under open flap conditions.

High scores were also achieved in a study by Rühling and co-workers (22), who studied the subgingival scaling performance of untrained operators in six 2-hour lessons during a 10- week training program. The subjects were divided into two groups of 11 participants each, to perform subgingival scaling either with Gracey curettes or a power-driven inserts with diamond coating on typodont models. At baseline the Gracey group achieved $63.1 \%$ vs. $52.3 \%$ removal in the power-driven group. After a training period of 9 and 11 weeks, the operators reached $84.7 \%$ in the Gracey group and $81.3 \%$ in the power-driven group. The test teeth also involved more complicated root structures. Nevertheless, this study group started with higher baseline scores and was retested after 12-hours of practical training. Both this study and our current study showed acceptable results and improvements during a course with limited instruction and practice time.

The materials and study design used in our study comes with some notable limitations. First of all, working on mannequin heads does not mimic a true clinical setting with human patients. We did not attempt to control for age or gender of the 
dental students, nor did we screen the students to determine any possible prior dental experience. We do assume that this was not an important factor in our study, as the dental students' baseline scores were similar to those of the lay people. Accordingly, because the positive control group, experienced dental hygienists, were not more proficient than the dental students after 8.5 hours of practice, it may be assumed that their lack of recent experience working on plastic teeth played into the results they were able to obtain. If the dental hygienists had been given practice time on the typodonts, immediately prior to completing the 5-minute exercise, their results might conceivably have been much better. If all participants, including the lay people, had the same amount of time to practice on the typodonts, the end results might likely have been the same for all. Finally, this study only evaluated the outcome of debriding one single-rooted tooth. The results therefore offer limited insight on the overall ability of the students and fail to show performance on areas with limited access and difficult anatomy.

Concerning the acquisition of dental skills, a few interesting questions remain: How do students learn manual dexterity? How much time is required to acquire the manual skills needed to perform dental procedures? Are pre-clinical courses (work on plastic teeth) a sufficient preparation for performing clinical procedures on patients? Previous studies have shown that manual dexterity skills can be acquired during dental curricular practice (16), if not the exact mechanism. Plastic teeth clearly do not provide a "life-like" practice environment, but the alternatives (extracted teeth or hepatic systems) present unsolved ethical, physical and technical dilemmas. Since students have been traditionally taught clinical skills on plastic teeth and have successfully gone on to treat patients in their schools' clinics, this method, even if not optimal, has stood the test of time. That makes the final question the most salient; how might students be encouraged to reach their optimal level of clinical proficiency? 
Two methods mentioned in the literature include motivational learning models and encouragement. A review of non-traditional evaluation models that included "nongraded" assessments, discussed a higher performance due to less stress and fatigue of the students. A normative grading system, compared to a traditional numerical grading system, also proved to be more accepted by the students and appeared to show better interaction with the clinical faculty (23). The results of our study have shown that the learning of pre-clinical skills may not require the amount of time that was traditionally provided for in earlier dental school curricula. However not all students were able to reach the same level of proficiency in the time provided. Therefore, more work is needed to determine methods that will help students who are initially weaker in their manual skills to progress faster and more reliably. Also maintaining long-term quality is an important factor to consider.

\section{Conclusion}

A two-week course consisting of only 8.5 hours in periodontal pre-clinical scaling practice was sufficient - within the limitations of the present study - to allow undergraduate dental students to debride a maxillary canine in a typodont model to a level similar to that of experienced dental hygienists and for some students to reach a level close to that of the reference tooth scaled under direct vision.

\section{Acknowledgment}

The present study was undertaken without external funding. All authors state that they have no conflict of interest. 


\section{Disclosure}

There is no financial, economic or professional interest that may have influenced the study by any of the researchers involved. 


\section{Reference}

1. http://www.eua.be/policy-representation/higher-education-policies/the-europeanhigher-education-area-and-the-bologna-process

2. Sanz M. Dental Education and the Bologna Process. Eur J Dent Educ. 2003;7:143-146.

3. R Core Team (2015). R: A language and environment for statistical computing. $R$ Foundation for Statistical Computing, Vienna, Austria. URL: https://http://www.R-project.org/.

4. Pohlert T (2014). The Pairwise Multiple Comparison of Mean Ranks Package (PMCMR). R package, URL: http://CRAN.R-project.org/package=PMCMR.

5. Carroll, A.M. and G.M. Schuster, Correlation Between Students' Dental Admission Test Scores and Performance on a Dental School's Competency Exam. J Dent Educ. 2015;79(11):1325-1329.

6. Gray, S.A. and L.P. Deem, Predicting student performance in preclinical technique courses using the theory of ability determinants of skilled performance. J Dent Educ. 2002;66(6):721-727 .

7. Kramer, G.A., Value in dental aptitude testing for minority applicants. J Dent Educ. 1999;63(10):759-65.

8. Graham, J.W., Substitution of perceptual-motor ability test for chalk carving in Dental Admission Testing Program. J Dent Educ. 1972;36(11):9-14.

9. Kao, E.C., et al., Wire-bending test as a predictor of preclinical performance by dental students. Percept Mot Skills. 1990;71(2):667-73.

10. Kothe, C., J. Hissbach, and W. Hampe, Prediction of practical performance in preclinical laboratory courses - the return of wire bending for admission of dental students in Hamburg. GMS Z Med Ausbild. 2014;31(2):Doc22.

11. Walcott, A.M., G.W. Knight, and R.E. Charlick, Waxing tests as predictors of students' performance in preclinical dentistry. J Dent Educ. 1986;50(12):716-21.

12. Suksudaj, N., et al., Acquiring psychomotor skills in operative dentistry: do innate ability and motivation matter? Eur J Dent Educ. 2012;16(1):e187-94.

13. Lundergan, W.P., E.J. Soderstrom, and D.W. Chambers, Tweezer dexterity aptitude of dental students. J Dent Educ. 2007;71(8):1090-7.

14. Luck, O., B. Reitemeier, and K. Scheuch, Testing of fine motor skills in dental students. Eur J Dent Educ. 2000;4(1):10-4. 
15. Boyle, A.M. and J.C. Santelli, Assessing psychomotor skills: the role of the Crawford Small Parts Dexterity Test as a screening instrument. J Dent Educ. 1986;50(3):176-9.

16. Ranney, R.R., M.B. Wilson, and R.B. Bennett, Evaluation of applicants to predoctoral dental education programs: review of the literature. J Dent Educ. 2005;69(10):1095-106.

17. Giuliani, M., et al., Is manual dexterity essential in the selection of dental students? Br Dent J. 2007;203(3):149-55.

18. Brayer, W.K., et al., Scaling and root planing effectiveness: the effect of root surface access and operator experience. J Periodontol. 1989;60(1):67-72.

19. Fleischer, H.C., et al., Scaling and root planing efficacy in multirooted teeth. J Periodontol. 1989;60(7):402-9.

20. Kocher, T., D. Riedel, and H.C. Plagmann, Debridement by operators with varying degrees of experience: a comparative study on manikins. Quintessence Int. 1997;28(3):191-6.

21. Rühling, A., et al., Learning root debridement with curettes and power-driven instruments. Part II: Clinical results following mechanical, nonsurgical therapy. J Clin Periodontol. 2003;30(7):611-5.

22. Rühling, A., et al., Learning root debridement with curettes and power-driven instruments. Part I: a training program to increase effectivity. J Clin Periodontol. 2002;29(7):622-9.

23. Hauser, A.M. and D.M. Bowen, Primer on preclinical instruction and evaluation. J Dent Educ. 2009;73(3):390-8. 
Figure 1. Student-scaled teeth, after removal from the typodont models 
Figure 2. Maxillary left canine in the typodont model.

A: typodont model with gingiva mask; B: before lacquer removal; C: after lacquer removal (reference tooth, scaled under direct vision) 
Figure 3. Box-plot graph presenting the mean values, standard deviation, median values, interquartile ranges, minimum and maximum of cleaned tooth surfaces achieved by the student groups of each year tested, before and after training, and their comparison with the positive (experienced dental hygienists \& reference tooth) and negative (laypeople) control groups. Statistically significant differences are indicated with differing capital letters. 
Table 4. Mean and standard deviation of each class year and participants 
Table 5. Pairwise comparisons between the participants ( $p-$ values), using Conover's posthoc test with Holm adjustment for multiple testing. 
Figure 1.

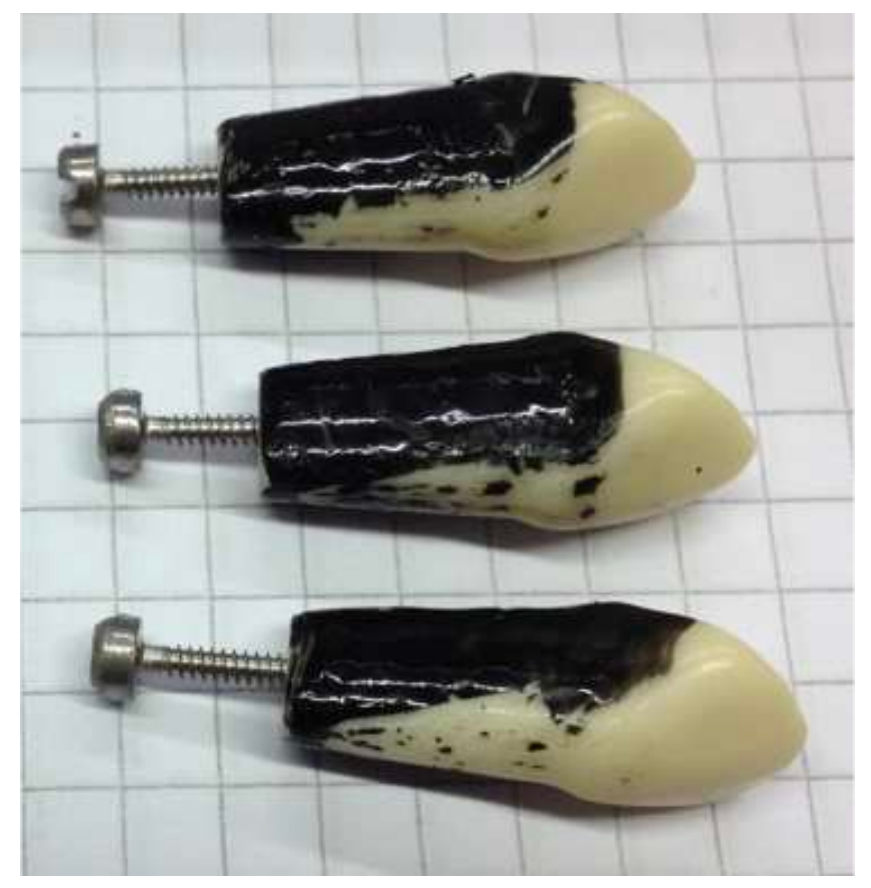


Figure 2. 
Figure 3 .

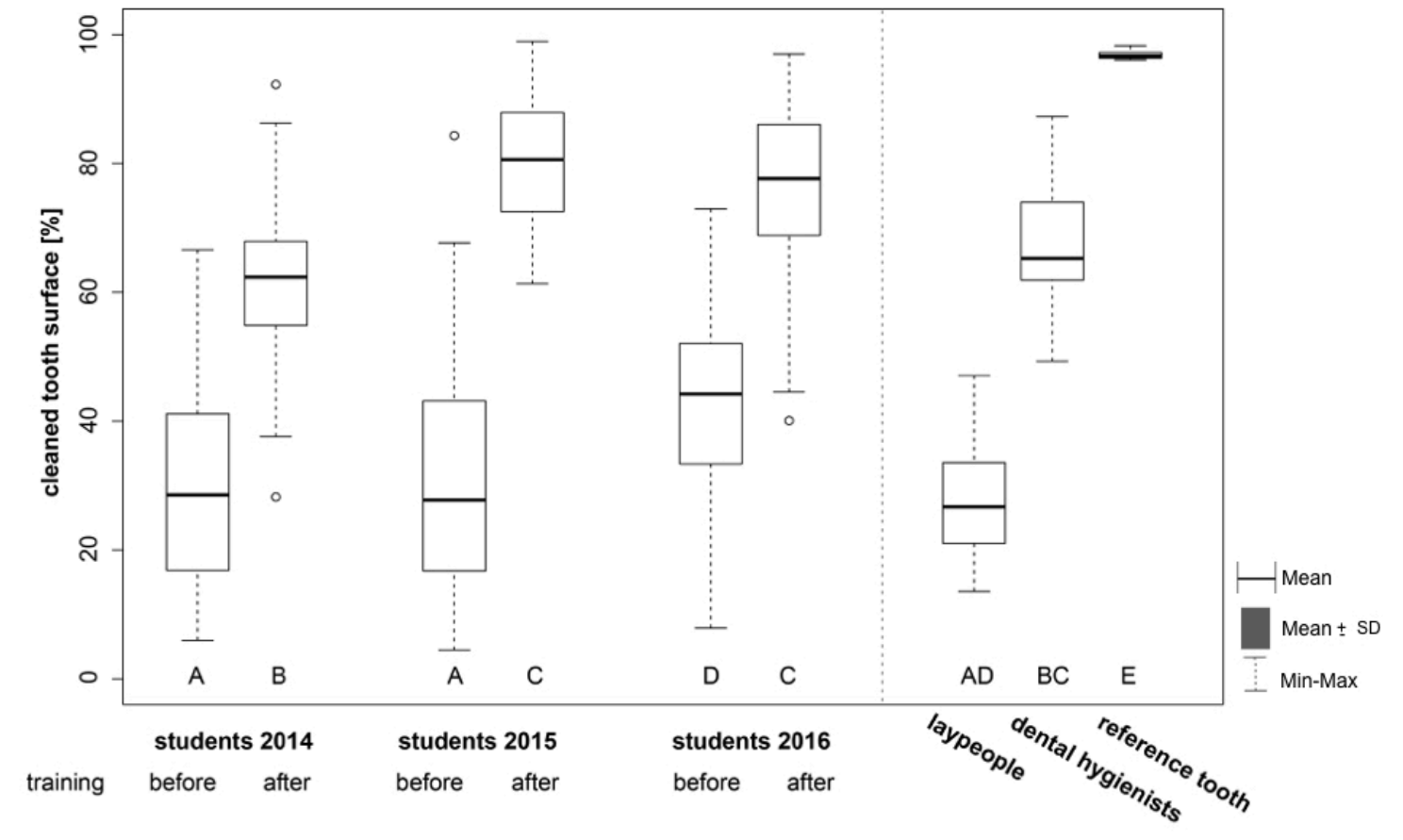



Table 4.

\begin{tabular}{|ll|l|l|l|}
\multicolumn{1}{c}{} & \multicolumn{1}{c}{ n } & \multicolumn{1}{c}{ SD } \\
\hline Students 2014 & before & 41 & 29.85 & 15.26 \\
Students 2014 & after & 41 & 61.68 & 12.18 \\
Students 2015 & before & 34 & 31.00 & 19.31 \\
Students 2015 & after & 34 & 79.46 & 10.45 \\
Students 2016 & before & 48 & 42.11 & 15.80 \\
Students 2016 & after & 48 & 76.08 & 13.39 \\
Laypeople & & 8 & 27.89 & 10.47 \\
Dental Hygienists & 8 & 67.36 & 11.40 \\
Reference Tooth & 10 & 96.87 & 00.70 \\
\hline
\end{tabular}

$\mathrm{n}=$ number in the group, $\mathrm{M}=$ Mean, $\mathrm{SD}=$ standard deviation 
Table 5.

\begin{tabular}{|c|c|c|c|c|c|c|c|c|c|}
\hline & & $\begin{array}{c}\text { Students } \\
2014 \\
\text { before } \\
\end{array}$ & $\begin{array}{c}\text { Students } \\
2014 \\
\text { after } \\
\end{array}$ & $\begin{array}{c}\text { Students } \\
2015 \\
\text { before } \\
\end{array}$ & $\begin{array}{c}\text { Students } \\
2015 \\
\text { after }\end{array}$ & $\begin{array}{c}\text { Students } \\
2016 \\
\text { before }\end{array}$ & $\begin{array}{c}\text { Students } \\
2016 \\
\text { after }\end{array}$ & Laypeople & $\begin{array}{c}\text { Dental } \\
\text { Hygienists }\end{array}$ \\
\hline Students 2014 & after & $p<0.001$ & & & & & & & \\
\hline Students 2015 & before & $p=1$ & $p<0.001$ & & & & & & \\
\hline Students 2015 & after & $p<0.001$ & $p<0.001$ & $p<0.001$ & & & & & \\
\hline Students 2016 & before & $p=0.002$ & $p<0.001$ & $p=0.02$ & $p<0,001$ & & & & \\
\hline Students 2016 & after & $p<0.001$ & $p<0.001$ & $p<0.001$ & $p=1$ & $p<0.001$ & & & \\
\hline Laypeople & & $p=1$ & $p<0.001$ & $p=1$ & $p<0.001$ & $p=0.1$ & $p<0.001$ & & \\
\hline Dental Hygienists & & $p<0.001$ & $p=1$ & $p<0.001$ & $p=0.12$ & $p<0.001$ & $p=0.43$ & $p<0.001$ & \\
\hline Reference Tooth & & $p<0.001$ & $p<0.001$ & $p<0.001$ & $p=0.01$ & $p<0.001$ & $p<0.001$ & $p<0.001$ & $p<0.001$ \\
\hline
\end{tabular}

